\title{
A REVIEW OF ONGOING ITU-R STUDIES TO ALLOW SHIPBOARD SATCOM SYSTEMS TO USE FREQUENCY BANDS SHARED BY THE FIXED SATELLITE AND FIXED SERVICES
}

\author{
Gregory A. Bostrom \\ Roy A. Axford, Jr. \\ Space \& Naval Warfare Systems Center \\ Signals Technology Branch (D841) \\ San Diego, California \\ gbostrom@spawar.navy.mil \\ axfordra@spawar.navy.mil
}

\begin{abstract}
The U.S. Navy (USN) Challenge Athena (CA) program, which provides two-way shipboard satellite communications (SATCOM) at data rates up to $1.544 \mathrm{Mbps}$ (T1), utilizes the C-band frequencies: 5,925-6,425 MHz earth-tospace and 3,700-4,200 MHz space-to-earth. The International Telecommunication Union (ITU) has allocated $C$ band for the Fixed Satellite Service (FSS) and the point-topoint, line-of-sight microwave links of the Fixed Service (FS) on a co-primary basis throughout the world. CA is best described as a broadband Maritime Mobile Satellite Service (MMSS), a subset of the Mobile Services (MS). However, there is no ITU MS, let alone MMSS, allocation in C-band. Therefore, from a regulatory perspective, $C A$ 's operation must be done on a not-to-interfere basis, without protection from electromagnetic interference (EMI) caused by incumbent users of the band (i.e., the FS and FSS). But the USN is not alone with requirements for broadband services that must be provided throughout the oceans. Earth stations on vessels ("ESVS" as they are referred to in the ITU) are also widely used by the cruise ship and offshore oil industries. Therefore, delegates representing several broadband maritime SATCOM service providers are cooperating with FS delegates in ITU Radiocommunication Bureau (ITU-R) Working Party 4-9S to study the potential for interference from ESVS and to develop ITU-R Recommendations for ESV use of FS/FSS shared frequency bands. This paper discusses WP 4-9S' ESV-on-FS interference avoidance studies, which are currently being conducted in preparation for World Radiocommunication Conference 2003 (WRC-2003). The methods described herein could be adapted to other situations where Mobile Satellite Service (MSS) transmissions might interfere with FS and FSS systems.
\end{abstract}

\section{BACKGROUND}

World Radiocommunication Conference 1997 (WRC1997) recognized the need for technical studies of the potential for interference with the terrestrial line-of-sight microwave links of the Fixed Service (FS) from earth stations on vessels (ESVs) operating in Fixed Satellite Service
(FSS) frequency bands shared by the FS. WRC-1997 formulated WRC-2000 Agenda Item 1.8: "To consider regulatory and technical provisions to enable earth stations located on board vessels to operate in the fixed-satellite service networks in the bands 3,700-4,200 $\mathrm{MHz}$ and $5,925-6,425 \mathrm{MHz}$, including their coordination with other services allocated in these bands." The group assigned to this topic continues to be International Telecommunication Union Radiocommunication Bureau (ITU-R) Working Party 4-9S (WP 4-9S), which studies technical issues in FS/FSS frequency sharing.

Although progress was made during the study period between WRC-1997 and WRC-2000, WP 4-9S was not able to complete Draft New Recommendations (DNRs) on ESV operations in time for consideration by WRC-2000. Therefore, the work was continued into the inter WRC2000 - WRC-2003 study period. The corresponding WRC2003 Agenda Item (No. 1.26) is: "To consider the provisions under which earth stations located on board vessels could operate in the fixed-satellite service networks, taking into account the ITU-R studies in response to Resolution 82 (WRC-2000)." Whereas WRC-2000 specifically dealt with C-band ESVs, WRC-2003 Agenda Item 1.26 only limits the considered bands to FSS allocations. WP 4-9S is considering $\mathrm{Ku}$-band in addition to $\mathrm{C}$-band as it continues its work on ESV DNRs in preparation for WRC-2003.

\section{ESV REGULATORY STATUS}

The ITU has allocated the 4 and $6 \mathrm{GHz}$ bands (C-band) for the FS and the FSS throughout the world. The FS and FSS share these bands on a co-primary basis. Maritime use of a broadband FSS-like service is best described as a Maritime Mobile Satellite Service (MMSS), a subset of the Mobile Services (MS). However, there is no ITU MS, let alone MMSS, allocation in $4 / 6 \mathrm{GHz}$. (The same is true in the $12 / 14 \mathrm{GHz}$ Ku-bands.) In spite of this, ESVs may operate in C-band FSS networks under Section S4.4 of the Radio Regulations as long as they do not claim protection from, nor cause interference to, other services having allocations in the band [1]. In fact, there is already widespread use of ESVs in C-band by the U.S. Navy (USN) and the cruise ship and offshore oil industries. C-band is attractive 
for providing wideband SATCOM services to maritime customers because it offers global coverage with multiple 36- $\mathrm{MHz}$ wide transponders. (Depending on its transmitter power and receive figure-of-merit, each $36-\mathrm{MHz}$ wide $\mathrm{C}$ band transponder typically supports three to four maritime full-duplex T1 links.) Although L-band offers global coverage with an MMSS allocation, the available per-channel bandwidth at L-band is currently inadequate to provide the full range of broadband services increasingly demanded by the maritime market. By agreeing to study ways in which these services can be provided to maritime customers via other bands (e.g., C- and Ku-band) without causing unacceptable interference to incumbent users, the ITU implicitly recognizes that there is currently no other way to entirely satisfy ocean-going broadband communications needs.

\section{ESV-ON-FS INTERFERENCE AVOIDANCE: THREE CASES}

The work on ESV-on-FS interference avoidance has been partitioned into three cases as discussed in the following subsections.

\section{Case 1: Ship is Stationary at a Pier In Port}

It has been proposed that the piers at which ESVequipped ships frequently dock could themselves be coordinated as FSS sites using existing methods. Since this is largely a regulatory question, WP 4-9S has not considered it.

In the U.S., an application to the Federal Communications Commission (FCC) by a domestic wideband maritime SATCOM provider to coordinate 17 frequently used U.S. piers as fixed FSS sites was rejected on 28 September 2000. However, in early February 2001, the National Telecommunications and Information Administration (NTIA) and the FCC appeared to have reached a preliminary agreement to treat pierside ESVs as FSS earth stations. Nonetheless, an agreement on this approach to regulating ESVs when they are pierside has not been finalized.

\section{Case 2: Ship is Underway, Beyond a Distance $d_{x x x}$ from the Coastline}

Given a description of ESV transmitted power and antenna pattern, FS receiver (FSR) sensitivity to interference, and the radioclimate and intervening terrain, there is a seaward distance $d_{x x x}$ from the coastline beyond which the probability of an ESV causing unacceptable interference to the FS ashore is negligible. If an ESV-equipped ship's operations do not bring it any closer than $d_{x x x}$ to the coastline, then coordination with the FS ashore is not necessary. Coordination is a financially costly activity in which the ESV operator contacts all FS operators in the coastal regions in question to determine which shared FS/FSS earth-to-space frequencies are unused by potentially affected FS stations. ESV transmissions within $d_{x x x}$ of the coast would necessarily be limited to those frequencies.
WP 4-9S has agreed on a manner for calculating $d_{x x x}$ and this is presented below. The resulting value of $d_{x x x}$ depends strongly on the values of many parameters. Depending on the interference criterion used to protect the FS, how often ESVs frequent the coastal region in question, the regional propagation characteristics, and other parameters, the calculated value of $d_{x x x}$ can range from 0 to $535 \mathrm{~km}$ in the $6 \mathrm{GHz}$ band. The authors have studied the sensitivity of the $d_{x x x}$ calculation result to most of the input parameters.

It is expected that a DNR for calculating $d_{x x x}$ will be completed prior to WRC-2003. This DNR will provide a general procedure that could be used at any frequency above roughly $1 \mathrm{GHz}$, with any ESV or FS system characteristics, in any region of the world. However, the DNR is also expected to provide fixed $d_{x x x}$ values for C-band and $\mathrm{Ku}$-band, which will necessarily be compromise values.

\section{Case 3: Ship is Underway, Within a Distance $d_{x x x}$ of the Coastline}

As discussed above, when the ship is underway on any path that brings it within $d_{x x x}$ of the coastline ${ }^{1}$, then it is recommended that the ESV operator coordinate with the FS ashore in order to avoid causing unacceptable interference. Work on a separate DNR for these coordinations is also in progress in WP 4-9S, but beyond the scope of this paper.

\section{IV. $d_{x x x}$ CALCULATION SETUP}

WP 4-9S has agreed on a setup for calculating $d_{x x x}$, which is shown in Figures 1 and 2. It is a worst case scenario in which the moving ESV is transmitting to a satellite at a low elevation angle in the same azimuthal direction as the FS Receiver (FSR) when the ship's path intersects the FSR antenna's boresight vector.

\section{INTERFERENCE CRITERIA}

How much interference a potential victim system can tolerate is defined with interference criteria. An interference criterion consists of two numbers:

- a permissible interference power level $I_{\max }$, which is often specified via an $I / N$ ratio (a receiver with a higher

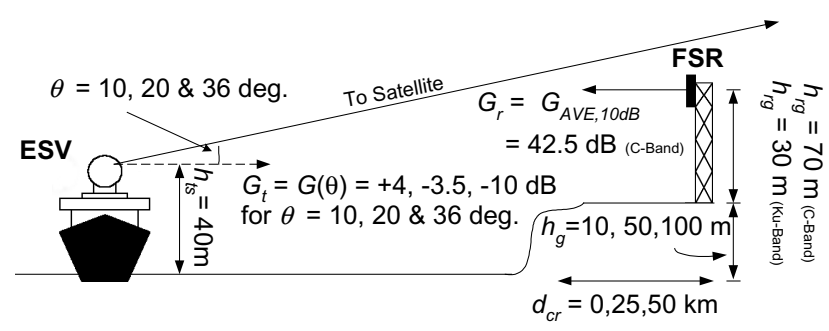

Figure 1 WP 4-9S ESV $d_{x x x}$ calculation setup.

\footnotetext{
${ }^{1}$ Leaving or entering port, or transiting near the coast.
} 


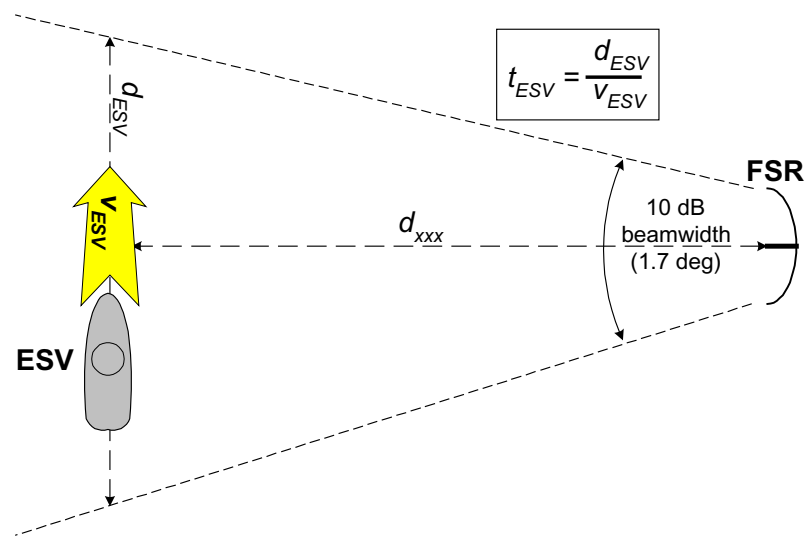

Figure 2 WP 4-9S ESV $d_{x x x}$ calculation setup, plan view.

level of thermal noise can tolerate more interference before any degradation in communications performance is incurred) and

- an amount of time during which it is tolerable for the permissible interference power level to be exceeded, often expressed as an annual percentage $p_{s}$.

Different interference criteria may be specified for various levels of degraded performance. For protecting the FS from ESVs in the 6-GHz band, ITU-R WP $9 \mathrm{~A}^{2}$ has derived two interference criteria [2]:

- $I / N=23 \mathrm{~dB}$ not to be exceeded for more than $p_{s}=$ $1.2 \times 10^{-5} \%$ of the time for the severely errored seconds (SES) level.

- $\quad I / N=19 \mathrm{~dB}$ not to be exceeded for more than $p_{s}=$ $4.5 \times 10^{-4} \%$ of the time, errored seconds (ES) level.

The time percentages above are annualized values corresponding to 1.6 seconds and 141.9 seconds per year, respectively. Note that, as is generally the case, the higher interference level is tolerable for a shorter time.

The value of $I_{\max }$ is calculated as follows:

$$
I_{\max }=\left(\frac{I}{N}\right)_{I C}+10 \log _{10}\left(k T_{F S R} B_{F S R}\right)(\mathrm{dBW})
$$

where

$$
\begin{aligned}
\left(\frac{I}{N}\right)_{I C}: & \text { defined in interference criterion }(\mathrm{dB}), \\
k: & \text { Boltzman's constant }(\mathrm{W} / \mathrm{K} / \mathrm{Hz}), \\
T_{F S R}: & \text { system noise temperature of the FSR }(\mathrm{K}), \\
B_{F S R}: & \text { occupied bandwidth of FSR }(\mathrm{Hz}) .
\end{aligned}
$$

\footnotetext{
${ }^{2}$ ITU-R Working Party 9A studies FS performance and availability, interference objectives and analysis, effects of propagation and terminology. They are FS subject matter experts.
}

\section{MINIMUM REQUIRED BASIC TRANSMISSION LOSS}

Based on the power the ESV radiates in the direction of the FSR and $I_{\max }$, the minimum required basic transmission loss is calculated as follows:

$$
L_{b, \text { min }}=P_{t, \text { max }}+G_{t}+G_{r, \text { ave }}-I_{\text {max }}-L_{F S R \text { feed }}
$$

where

$L_{b, \min }: \quad$ minimum required basic transmission loss $(\mathrm{dB})$,

$P_{t, \max }:$ maximum transmit power at the ESV antenna input flange (dBW),

$G_{t}$ : $\quad$ ESV antenna gain in the direction of the FS receiver (FSR) ( $\mathrm{dBi})$,

$G_{r, a v e}:$ average gain of the FSR antenna within a beamwidth of interest. WP 4-9S uses the -10 $\mathrm{dB}$ beamwidth ( $\mathrm{dBi})$ [3],

$L_{F S R \text { feed }}$ : loss in the feed from the FSR antenna aperture to the low noise amplifier $(\mathrm{dB})$.

The annual time percentage $p_{s}$ during which it is tolerable for $I_{\max }$ to be exceeded is equal to the annual time percentage during which it is permissible for $L_{b, \min }$ to not be exceeded. Occurrences of propagation phenomena that allow enhanced electromagnetic coupling (reduced transmission loss) over the horizon above roughly $1 \mathrm{GHz}$ are rare (more rare as the distance from the center point of the propagation path to the equator increases). A lower $p_{s}$ implies that the rarer of these enhanced levels of coupling are being considered, and therefore that a greater distance $d_{x x x}$ between the interference source (ESV) and the potential victim (FSR) is required in order to achieve $L_{b, \text { min }}$.

The available ITU-R Recommendations for calculating the required separation distance between FSS and FS stations in order to satisfy interference criteria are Recs. ITUR P.452 [4] and P.620 [5]. WP 4-9S uses both in the ESV interference avoidance studies.

\section{ITERATIVE $d_{x x x}$ CALCULATION PROCEDURE}

Interference from an ESV cannot occur unless the ESV is present. Because the ESV is not always present, it is not appropriate to use $p_{s}$ directly as the time percentage for which the required minimum transmission loss is not exceeded propagation model input parameter (i.e., $p$ in ITU-R Recs. P.452 and P.620). To do so would be equivalent to assuming that the passing of an ESV always coincides with the occurrence of the rarest propagation conditions. The passing of an ESV is statistically independent of propagation conditions. Therefore

$$
p=\left(p_{s} / p_{E S V}\right) \times 100 \%
$$

where 
$p_{E S V}:$ the annual percentage of time during which ESVs are present.

Clearly, $p$, through $p_{E S V}$, depends on how often an ESV passes through the FSR -10-dB beamwidth (WP 4-9S quantifies this as the number of ESV passes per year, $f_{E S V}$ ) and on how much time the ESV spends in this beamwidth

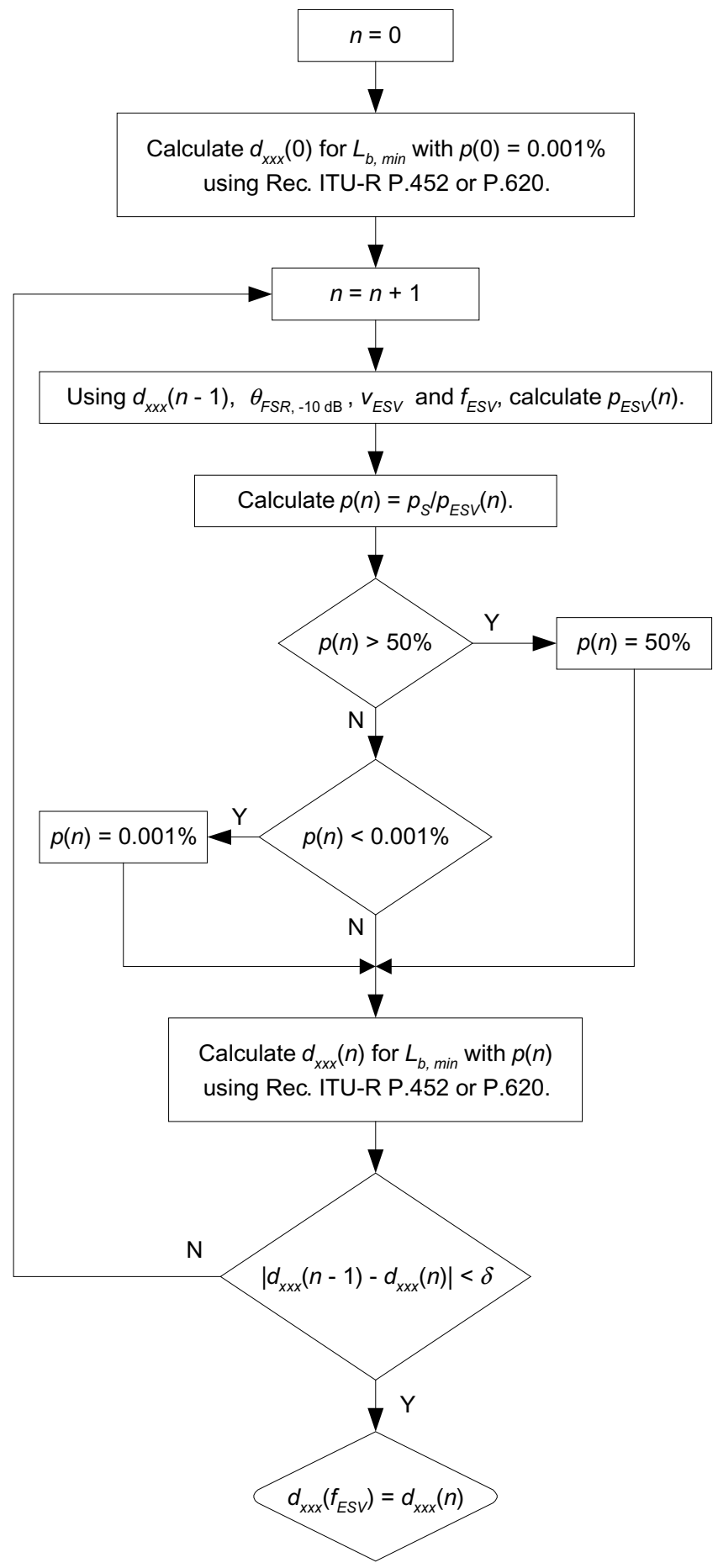

Figure 3 Iterative procedure for calculating $d_{x x x}\left(f_{E S V}\right)$. during a pass. But this amount of time depends on the distance from the ESV to the FSR (see Figure 2). Since $p$ depends on this distance and vice versa, an iterative method for determining the minimum $d_{x x x}$ that satisfies an interference criterion is unavoidable.

Figure 3 presents a flow chart detailing the iterative $d_{x x x}$ calculation procedure that is carried out for each desired value of $f_{E S V}$, the range of which is 1 to 1000 in WP 49S' work thus far. Regardless of $f_{E S V}$, the procedure is initiated under the assumption that the ESV is always present, yielding $d_{x x x}(0)$. The next iteration determines how much time the ESV would spend in the $-10-\mathrm{dB}$ beamwidth of the FSR at the distance $d_{x x x}(0)$ and then calculates $d_{x x x}(1)$ based on the resulting value of $p$. The procedure continues until the difference between the $d_{x x x}$ results on successive iterations is less than a threshold $\delta=1 \mathrm{~km}$. There is no basis for using $p<0.001 \%$ (annual percentage equivalent to 5.25 minutes per year) in the propagation models in Recs. ITUR P.452 and P.620 [6]. The procedure depicted in Figure 3 observes this restriction.

\section{6-GHz $d_{x x x}$ SENSITIVITY STUDY}

We have studied the sensitivity of the $6-\mathrm{GHz} d_{x x x}$ results to several input parameters. The propagation losses were calculated using the methods of ITU-R P.452-9 with a computer code that was verified by a WP 4-9S correspondence group. The input parameters and values used in the study are shown in Table 1.

Table $16-\mathrm{GHz} d_{x x x}$ sensitivity study input parameters and values.

\begin{tabular}{|l|l|}
\hline Interference Criterion & $\begin{array}{c}\mathrm{ES}\left(I / N=19 \mathrm{~dB}, p_{s}=4.5 \times 10^{-4} \%\right) \\
\mathrm{SES}\left(I / N=23 \mathrm{~dB}, p_{s}=1.2 \times 10^{-5} \%\right)\end{array}$ \\
\hline $\begin{array}{l}\text { Latitude, } \phi \text { (with repre- } \\
\text { sentative radioclimatic } \\
\text { parameters) }\end{array}$ & $\begin{array}{c}0(N=375, \Delta N=85) \\
20(N=350, \Delta N=70) \\
50(N=320, \Delta N=50)\end{array}$ \\
\hline $\begin{array}{l}\text { ESV Antenna Elevation } \\
\text { Angle, } \theta\end{array}$ & $10,20,36$ degrees \\
\hline FSR distance to coast, $d_{c r}$ & $0,25,50 \mathrm{~km}$ \\
\hline Ground height, $h_{g}$ & $\begin{array}{l}10,50,100 \text { meters above mean sea } \\
l e v e l\end{array}$ \\
\hline Ship's Speed, $v_{E S V}$ & $5,10,20 \mathrm{knots}$ \\
\hline Number of passes/yr., $f_{E S V}$ & $\begin{array}{l}1,2,5,12,30,60,120,180,240, \\
365,730,1000\end{array}$ \\
\hline
\end{tabular}

The sensitivity study results support the following conclusions.

1. The $d_{x x x}$ results are most sensitive to latitude $\phi$ (via the latitude-dependent radioclimatic parameters), the ESV elevation angle $\theta$ and the annual number of passes $f_{E S V}$.

2. Varying the ESV's speed $v_{E S V}$ from 5 to 20 knots causes 50 to $100 \mathrm{~km}$ of variation in $d_{x x x}$ for a low number of passes $\left(f_{E S V} \leq 10\right)$ and 25 to $30 \mathrm{~km}$ of variation 
at the higher numbers of passes $\left(f_{E S V} \geq 200\right)$. Beyond that, however, not much insight is gained by varying $v_{E S V}$ and it is probably just as well to use a single speed of 10 knots.

3. Varying the ground height $h_{g}$ from 10 to 100 meters typically causes a change in $d_{x x x}$ that is less than $25 \mathrm{~km}$. However, in some cases where $d_{x x x} \approx 100$ to $200 \mathrm{~km}$ for $h_{g}=10$ meters and $\phi \geq 20$ degrees, increasing $h_{g}$ to 100 meters results in shadowing that reduces $d_{x x x}$ to 0 $\mathrm{km}$ for $f_{E S V}<10$. This drastic reduction of $d_{x x x}$ only occurs when the FSR is inland from the coast, i.e., $d_{c r}=$ 25 or $50 \mathrm{~km}$. Furthermore, for all latitudes, the reduction in $d_{x x x}$ resulting from increasing $h_{g}$ becomes insignificant as $f_{E S V}$ approaches 1000 passes per year.

4. Moving the FSR inland (increasing $d_{c r}$ from 0 to 25 and $50 \mathrm{~km}$ ) decreases $d_{x x x}$ for $f_{E S V}<10$, especially at the higher latitudes. This is true not just because $d_{x x x}$ is reduced to a portion of the ESV-FSR separation distance for $d_{c r}>0$ (whereas when $d_{c r}=0, d_{x x x}$ is the entire ESV-FSR separation distance). Reduced occurrence of anomalous propagation phenomena over land (as compared to over water) also helps to reduce $d_{x x x}$ as $d_{c r}$ increases. However, for all latitudes, the reduction in $d_{x x x}$ resulting from increasing $d_{c r}$ becomes insignificant as $f_{E S V}$ approaches 1000 passes per year.

\section{SUMMARY AND CONCLUSIONS}

Considering the vast economic investment in FS infrastructure, the reaction to ESVs by FS delegates has been very cautious. This assertion is supported by comparing WP 9A's interference criteria for protecting the FS from ESVs (see Section V above) with a more established one used when traditional FSS stations coordinate with the FS. The interference criterion for FSS-FS coordination at Cband given in [7] is:

- $I / N=37 \mathrm{~dB}$ not to be exceeded for more than $p_{s}=$ $2.5 \times 10^{-3} \%$.

For a given FSR, this Appendix S7 criterion yields an $I_{\max }$ value that is 14 and $18 \mathrm{~dB}$ greater than the SES and ES levels, respectively, defined by WP 9A. The Appendix S7 $p_{s}$ is 208 and 5.5 times longer than WP 9A's corresponding SES and ES values, respectively.

Furthermore, although the $d_{x x x}$ calculation setup is the best compromise between analytical tractability and realism that WP 4-9S has been able to reach, it represents an extraordinary worst case. Because the typical length of a 6GHz FS link is $25 \mathrm{~km}$, when $d_{c r}<25 \mathrm{~km}$, WP 4-9S' $d_{x x x}$ calculation setup is only realistically representative of situations in which the ESV-equipped ship would already be within $25 \mathrm{~km}$ of the shore. No responsible C-band ESV operator would dispute the need for coordination when operating within $25 \mathrm{~km}$ of a coast populated with FSRs facing seaward.
WP 4-9S' $d_{x x x}$ calculation technique produces results that are highly sensitive to the input parameters (especially $\phi, \theta$ and $f_{E S V}$ ). In many cases, the resulting $d_{x x x}$ values are overly conservative for application to situations more general than the calculation setup (i.e., situations in which FSRs are not pointed out to sea and the ESV is not tracking a satellite in the direction of an FSR). Nonetheless, the ESV-on-FS interference avoidance studies proceeding in WP 4-9S represent the best efforts of all participants to develop ITU-R Recommendations that will adequately protect the incumbents while allowing maritime access to broadband services via FSS frequency bands. Since a single globally representative set of parameters cannot be defined, any single $d_{x x x}$ value recommended by the WP 4-9S for a given frequency band will be an "administrative" distance reached via compromise.

\section{REFERENCES}

[1] International Telecommunication Union, Radiocommunication Bureau (ITU-R), Resolution 82, World Radiocommunication Conference 2000, Istanbul, Turkey, 1 May - 2 June 2000.

[2] ITU-R Working Party 9A, "Derivation of Fixed Service Interference Criteria in the $6 \mathrm{GHz}$ and $14 \mathrm{GHz}$ Bands," Liaison Statement to Working Party 4-9S, Document 49S/87, 19 March 2001.

[3] France, "Protection Distance for FSS Earth Stations On Board Vessels in the $4 / 6 \mathrm{GHz}$ Band," Input Contribution to Working Party 4-9S, Document 4-9S/133, 13 April 1999.

[4] ITU-R, Recommendation P.452-9, "Prediction Procedure for the Evaluation of Microwave Interference between Stations on the Surface of the Earth at Frequencies Above about $0.7 \mathrm{GHz}$," Geneva, Switzerland, 1999.

[5] ITU-R, Recommendation P.620-4, "Propagation Data Required for the Evaluation of Coordination Distances in the Frequency Range $100 \mathrm{MHz}$ to $105 \mathrm{GHz}$," Radiocommunication Bureau, Geneva, Switzerland, 1999.

[6] ITU-R Working Party $3 \mathrm{M}^{3}$, "Earth Stations Located On Board Vessels Operating in FSS Networks in Bands 3,7004,200 and 5,925-6,425 MHz," Liaison Statement to Working Party 4-9S, Document 4-9S/3, 27 July 2000.

[7] ITU-R, Radio Regulations, Appendix S7, "Methods for the Determination of the Coordination Area Around an Earth Station in Frequency Bands Between $100 \mathrm{MHz}$ and $105 \mathrm{GHz}, "$ Geneva, Switzerland, 2000.

\footnotetext{
${ }^{3}$ ITU-R Working Party 3M studies point-to-point and earthspace radiowave propagation. They are subject matter experts on the propagation models in Recs. ITU-R P.452 and P.620.
} 